\title{
ECOLOGICAL DIFFERENCES BETWEEN GENOTYPES IN A NATURAL POPULATION OF DAPHNIA MAGNA
}

\author{
PAUL D. N. HEBERT* \\ Department of Genetics, University of Cambridge, Cambridge
}

Received 12.ii.74

\begin{abstract}
SumMary
Large changes in genotypic frequencies have been observed over a 2-year period in a population of Daphnia magna polymorphic for two alleles of malate dehydrogenase. These changes were not the result of migration or random drift. Genotypic differences in both parthogenetic and sexual egg production were observed. There was a strong correlation between the parthogenetic fecundities of the genotypes and their frequencies 1 month later. Sexual egg production was enhanced among low fitness genotypes. These observations strongly suggest that populations of Daphnia magna consist of a limited number of ecologically differentiated clones.
\end{abstract}

\section{INTRODUCTION}

THE occurrence of genetic variation in primary fitness components has been well established in laboratory experiments (Dobzhansky, Lewontin and Pavlovsky, 1964; Sokal and Sonleitner, 1968). Studies on wild populations have been much more restricted because determination of characters such as fecundity or developmental rate ordinarily requires continuous monitoring of individuals. However, in natural populations of the cladoceran crustacean, Daphnia magna, differences in reproductive phenotype can be determined visually.

D. magna reproduces both parthenogenetically and sexually. When food is abundant, parthenogenetic eggs are released into the brood pouch after each moult of adult life. Embryogenesis occurs in the pouch and from two (at $20^{\circ}$ G.) to ten (at $8^{\circ}$ G.) days elapse before the young are released (MacArthur and Baillie, 1929). The number of young in a brood can vary from 1 to over 100 and is directly related to food availability. Indeed, when food levels are low, females become non-reproductive (Slobodkin, 1954). Normally the parthenogenetic offspring are female, but male progeny are produced if the adults are crowded. Sex determination is apparently environmental (Hebert and Ward, 1972). Parthenogenetic eggs which produce males are morphologically indistinguishable from female eggs. The stimulus for sexual egg production has not been clearly defined, although in the laboratory a rapid decline in food supply often leads to sexual egg formation (Slobodkin, 1954). Only two sexual eggs are produced at a time and they are easily distinguished from parthenogenetic eggs for they are enveloped by several membranes. These eggs normally diapause and in permanent habitats they often fail to hatch (Hebert, 1974). The same female can produce all three sorts of eggs and no sequential rigor is placed on this ability.

* Present address: School of Biological Sciences, University of Sydney, Sydney, 2006, Australia. 
Previous studies have shown that in permanent populations of $D$. magna genotypic frequencies are subject to violent, selectively determined oscillations and that gametic phase imbalance between loci is frequent (Hebert, 1974). Coupled with evidence of genotypic differences in reproductive characteristics (Hebert, Ward and Gibson, 1972), these observations suggest that Daphnia populations consist of a limited number of highly structured, ecologically differentiated genotypes.

The present study examines the relationship between ecological differences and changes of genotypic frequencies in a permanent population at Harlton. This population was studied because sampling was easy due to the small size of the pond and because the fauna was depauperate. Important predators of Daphnia such as fish, newts, watermites and Chaoborus larvae were absent. Other daphniids, with the rare exception of $D$. obtusa, were also absent.

\section{Methods of investigation}

Every 2 weeks, from May 1970 to June 1972, a sample of Daphnia was collected in the central area of the Harlton pond. Individuals in the sample were sorted into reproductive classes within an hour of collection and analysed, using disc electrophoresis. Detailed methods, including a description of the malate dehydrogenase $(\mathrm{MDH})$ variants mentioned in this paper,

TABLE 1

$M D H$ genotypic frequencies

\begin{tabular}{|c|c|c|c|c|}
\hline Date & $n$ & $\mathbf{M M}$ & MF & FF \\
\hline May 1970 & 188 & $0 \cdot 30$ & 0.47 & 0.23 \\
\hline June & 381 & $0 \cdot 18$ & 0.46 & 0.36 \\
\hline July & 310 & 0.09 & 0.43 & 0.48 \\
\hline August & $460 * *$ & 0.09 & 0.55 & 0.36 \\
\hline September & $294 * *$ & 0.05 & $0 \cdot 69$ & 0.26 \\
\hline October & $368 * *$ & 0.05 & 0.67 & $0 \cdot 28$ \\
\hline November & $284 * *$ & 0.04 & 0.71 & 0.25 \\
\hline December & $287 * *$ & $0 \cdot 04$ & 0.70 & 0.26 \\
\hline January 1971 & $306 * *$ & 0.04 & 0.65 & 0.31 \\
\hline February & $286 * *$ & 0.03 & 0.72 & 0.25 \\
\hline March & $288 * *$ & $0 \cdot 02$ & $0 \cdot 67$ & 0.31 \\
\hline April & $216 * *$ & 0.01 & 0.70 & 0.29 \\
\hline May & $297 * *$ & 0.01 & 0.74 & 0.25 \\
\hline June & $293 * *$ & 0.02 & 0.70 & 0.28 \\
\hline July & $144 * *$ & 0.01 & 0.78 & 0.21 \\
\hline August & $230 * *$ & 0.01 & $0 \cdot 68$ & $0 \cdot 31$ \\
\hline September & $272 * *$ & 0.01 & 0.71 & 0.28 \\
\hline October & $335 * *$ & 0.01 & 0.54 & 0.45 \\
\hline November & $240 * *$ & 0.01 & 0.46 & 0.53 \\
\hline December & $288 * *$ & 0.01 & 0.44 & 0.55 \\
\hline January 1972 & $288 * *$ & - & 0.40 & 0.60 \\
\hline February & $280 *$ & 0.01 & 0.36 & 0.63 \\
\hline March $\dagger$ & $144 *$ & - & $0 \cdot 36$ & 0.64 \\
\hline April & $288^{*}$ & - & 0.35 & 0.65 \\
\hline May & 291 & - & 0.20 & 0.80 \\
\hline June & 288 & - & 0.13 & 0.87 \\
\hline
\end{tabular}


have already been described (Hebert and Ward, 1972). The two samples analysed each month have been combined to produce a single estimate for each of the population parameters studied.

\section{Results}

\section{(i) $M D H$ frequencies}

During the first 5 months of sampling the frequencies of all three $\mathrm{MDH}$ genotypes changed markedly, but from October 1970 to September 1971 frequencies were comparatively stable. During the last 9 months of the study, however, a large increase in the frequency of the FF genotype was observed (Table 1).

To rule out the possibility of spatial heterogeneity of genotypic frequencies, additional samples were collected at opposite ends of the pond. On each occasion genotypic frequencies were similar at all of the sites (table 2).

TABLE 2

Spatial sampling

\begin{tabular}{|c|c|c|c|c|c|c|c|c|c|c|c|c|}
\hline \multirow[b]{2}{*}{ Date } & \multicolumn{4}{|c|}{ Site 1} & \multicolumn{4}{|c|}{ Site 2} & \multicolumn{4}{|c|}{ Site 3} \\
\hline & $n$ & MM & MF & $\mathrm{FF}$ & $n$ & MM & $\mathrm{MF}$ & $\mathrm{FF}$ & $n$ & $\mathrm{MM}$ & $\mathrm{MF}$ & $\mathrm{FF}$ \\
\hline $\begin{array}{l}23.7 .70 \\
7.10 .70\end{array}$ & $\begin{array}{r}143 \\
83\end{array}$ & $\begin{array}{l}0.13 \\
0.06\end{array}$ & $\begin{array}{l}0.46 \\
0.74\end{array}$ & $\begin{array}{l}0 \cdot 41 \\
0.20\end{array}$ & $\begin{array}{r}120 \\
72\end{array}$ & $\begin{array}{l}0.13 \\
0.06\end{array}$ & $\begin{array}{l}0.46 \\
0.72\end{array}$ & $\begin{array}{l}0.41 \\
0 \cdot 22\end{array}$ & $\begin{array}{r}120 \\
72\end{array}$ & $\begin{array}{l}0 \cdot 14 \\
0.03\end{array}$ & $\begin{array}{l}0.53 \\
0.71\end{array}$ & $\begin{array}{l}0.33 \\
0.26\end{array}$ \\
\hline $\begin{array}{r}1.6 .71 \\
1.9 .71\end{array}$ & $\begin{array}{l}149 \\
144\end{array}$ & $\begin{array}{l}0.02 \\
0 \cdot 01\end{array}$ & $\begin{array}{l}0.73 \\
0.73\end{array}$ & $\begin{array}{l}0.25 \\
0.26\end{array}$ & $\begin{array}{l}120 \\
120\end{array}$ & $\overline{0.01}$ & $\begin{array}{l}0 \cdot 70 \\
0 \cdot 75\end{array}$ & $\begin{array}{l}0.30 \\
0.24\end{array}$ & $\begin{array}{l}120 \\
120\end{array}$ & $\begin{array}{c}0.01 \\
-\end{array}$ & $\begin{array}{l}0.72 \\
0.68\end{array}$ & $\begin{array}{l}0.27 \\
0.32\end{array}$ \\
\hline
\end{tabular}

No direct estimates of population size were made, but it was evident that the Harlton population usually numbered many thousands of individuals. Subjective estimates indicated that population minima occurred during February-March of 1971 and 1972 and during late July of 1971.

\section{(ii) Reproductive status}

The first samples indicated that growth of the Harlton population was limited by a lack of food (table 3). Many of the females were without eggs and reproductive females carried small parthenogenetic broods or sexual eggs. From December 1970 until February 1971 almost all of the females were non-reproductive. Females had released their young in November and not produced new broods, perhaps because of a combination of low water temperatures and a scarcity of food. Reproduction began anew during February and broods remained large until the end of March, but by midApril they were much smaller and more of the females were without eggs. A sexual period was initiated at the end of May and extended until July. The almost total cessation of reproduction in early July suggested that the population had depleted its food supply. This was confirmed by analysis of lactate dehydrogenase phenotypes (Hebert, 1973) and indeed the population collapsed during late July. The survivors of the population crash produced large parthenogenetic broods during August and throughout the autumn. The increase in frequency of non-reproductive females from October until early December was apparently unrelated to food shortage as broods remained large. The subsequent decline in the frequency of 
non-reproductive females from late December until February 1972 was accompanied by an increase in brood size. Most females continued to carry large broods until mid-April, but in late April and May brood size declined, and more non-reproductive females were present. By late June most of the females were non-reproductive, but despite the evident deterioration in conditions sexual females remained rare.

TABLE 3

Reproductive status of the Harlton population

Females with parthenogenetic eggs

\begin{tabular}{|c|c|c|c|c|c|c|c|c|c|c|c|}
\hline & & & & & & & & & & & \\
\hline Date & females & $1-4$ & $5-9$ & $10-19$ & $20-39$ & $40-59$ & $60-79$ & 80-99 & $100-119$ & $\begin{array}{l}\text { Sexual } \\
\text { females }\end{array}$ & Male \\
\hline 5.8 .70 & 0.59 & 0.40 & 0.01 & - & - & - & - & - & - & - & _ \\
\hline 19.8 .70 & 0.57 & 0.41 & 0.02 & - & - & - & - & - & - & - & - \\
\hline 28.8.70 & 0.54 & $0 \cdot 21$ & $0 \cdot 25$ & - & - & - & - & - & - & - & - \\
\hline 8.9 .70 & $0 \cdot 63$ & 0.23 & $0 \cdot 14$ & - & - & - & - & - & - & - & - \\
\hline 30.9 .70 & $0 \cdot 40$ & 0.45 & $0 \cdot 14$ & - & 一 & - & - & - & - & 0.01 & + \\
\hline 7.10 .70 & 0.57 & $0 \cdot 33$ & $0 \cdot 09$ & - & - & - & - & - & - & 0.01 & + \\
\hline 14.10 .70 & $0 \cdot 72$ & $0 \cdot 13$ & $0 \cdot 05$ & - & - & - & - & - & - & $0 \cdot 10$ & + \\
\hline $28 \cdot 10 \cdot 70$ & 0.69 & $0 \cdot 13$ & $0 \cdot 10$ & - & - & - & - & - & - & 0.08 & + \\
\hline 12.11 .70 & 0.47 & $0 \cdot 03$ & 0.18 & 0.06 & - & - & - & - & - & $0 \cdot 26$ & + \\
\hline 23.11 .70 & 0.44 & $0 \cdot 01$ & $0 \cdot 26$ & $0 \cdot 08$ & - & - & - & - & - & 0.21 & + \\
\hline 9.12 .70 & $0 \cdot 80$ & 0.01 & 0.04 & - & - & - & - & & - & $0 \cdot 15$ & + \\
\hline 28.12.70 & 0.83 & 0.01 & 0.05 & 0.01 & - & - & - & - & - & $0 \cdot 10$ & + \\
\hline 6.1 .71 & $0 \cdot 85$ & 0.04 & $0 \cdot 04$ & 0.04 & - & - & - & - & - & 0.03 & 一 \\
\hline 27.1 .71 & 0.85 & 0.02 & $0 \cdot 05$ & $0 \cdot 07$ & $0 \cdot 01$ & - & - & - & - & - & - \\
\hline 5.2 .71 & 0.66 & 0.06 & $0 \cdot 10$ & $0 \cdot 15$ & 0.03 & - & - & - & - & - & - \\
\hline 19.2.71 & $0 \cdot 54$ & $0 \cdot 14$ & 0.07 & $0 \cdot 19$ & 0.06 & - & - & - & - & - & - \\
\hline 8.3 .71 & $0 \cdot 17$ & 0.03 & $0 \cdot 12$ & $0 \cdot 35$ & 0.33 & - & - & - & - & - & \\
\hline 31.3 .71 & 0.03 & 0.07 & 0.07 & 0.23 & $0 \cdot 37$ & $0 \cdot 19$ & 0.04 & - & - & - & - \\
\hline 19.4 .71 & 0.33 & 0.25 & $0 \cdot 38$ & 0.04 & - & - & - & - & - & - & - \\
\hline 27.4 .71 & 0.37 & 0.30 & 0.30 & 0.03 & - & - & - & - & - & - & 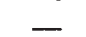 \\
\hline 11.5 .71 & $0 \cdot 18$ & $0 \cdot 16$ & $0 \cdot 61$ & 0.05 & - & - & - & - & - & - & - \\
\hline 27.5 .71 & $0 \cdot 10$ & 0.05 & $0 \cdot 61$ & 0.23 & - & - & - & - & - & 0.01 & + \\
\hline 6.6 .71 & $0 \cdot 26$ & 0.07 & $0 \cdot 51$ & 0.01 & - & - & - & - & - & $0 \cdot 15$ & + \\
\hline 21.6 .71 & $0 \cdot 40$ & $0 \cdot 20$ & $0 \cdot 19$ & - & - & - & - & - & - & 0.21 & + \\
\hline 6.7 .71 & 0.95 & 0.03 & - & - & - & - & - & - & - & 0.02 & + \\
\hline 8.8.71 & $0 \cdot 06$ & $0 \cdot 05$ & $0 \cdot 15$ & 0.53 & $0 \cdot 21$ & - & - & - & - & - & - \\
\hline 16.8 .71 & 0.07 & 0.01 & 0.01 & 0.18 & 0.56 & $0 \cdot 16$ & 0.01 & - & - & - & 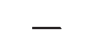 \\
\hline 1.9 .71 & $0 \cdot 11$ & $0 \cdot 05$ & $0 \cdot 30$ & 0.54 & - & - & - & - & - & - & - \\
\hline 23.9 .71 & 0.08 & $0 \cdot 14$ & 0.63 & $0 \cdot 15$ & - & - & - & - & - & - & - \\
\hline 14.10 .71 & $0 \cdot 20$ & 0.02 & $0 \cdot 05$ & $0 \cdot 40$ & $0 \cdot 32$ & 0.01 & - & - & - & - & - \\
\hline 25.10 .71 & 0.28 & 0.02 & 0.09 & $0 \cdot 22$ & $0 \cdot 34$ & 0.05 & - & - & - & - & - \\
\hline 15.11 .71 & 0.20 & 0.04 & 0.04 & 0.20 & 0.47 & 0.04 & $0 \cdot 01$ & - & - & - & - \\
\hline 24.11 .71 & $0 \cdot 28$ & $0 \cdot 01$ & 0.05 & $0 \cdot 17$ & 0.44 & 0.04 & 0.01 & - & - & - & - \\
\hline 7.12 .71 & 0.34 & $0 \cdot 01$ & $0 \cdot 09$ & $0 \cdot 17$ & $0 \cdot 35$ & $0 \cdot 04$ & - & - & - & - & - \\
\hline 29.12 .71 & $0 \cdot 28$ & $0 \cdot 01$ & $0 \cdot 01$ & 0.06 & $0 \cdot 39$ & $0 \cdot 17$ & 0.06 & 0.01 & - & 0.01 & - \\
\hline 17.1.72 & $0 \cdot 12$ & 0.01 & 0.01 & $0 \cdot 11$ & 0.45 & $0 \cdot 24$ & 0.04 & 0.01 & - & 0.01 & - \\
\hline 28.1.72 & 0.08 & 0.02 & $0 \cdot 01$ & $0 \cdot 10$ & $0 \cdot 56$ & $0 \cdot 20$ & $0 \cdot 02$ & 0.01 & - & - & - \\
\hline 14.2 .72 & 0.06 & 0.02 & 0.02 & 0.09 & 0.58 & 0.22 & 0.01 & - & - & - & - \\
\hline 24.2.72 & 0.08 & 0.02 & $0 \cdot 01$ & $0 \cdot 13$ & 0.55 & $0 \cdot 17$ & 0.03 & 0.01 & - & - & - \\
\hline 6.3 .72 & $0 \cdot 12$ & 0.01 & 0.01 & $0 \cdot 15$ & 0.57 & $0 \cdot 12$ & $0 \cdot 02$ & - & - & - & - \\
\hline 8.4 .72 & 0.01 & - & - & $0 \cdot 12$ & $0 \cdot 41$ & 0.22 & $0 \cdot 19$ & $0 \cdot 04$ & $0 \cdot 01$ & - & - \\
\hline 19.4.72 & $0 \cdot 24$ & $0 \cdot 16$ & 0.44 & $0 \cdot 12$ & 0.01 & 0.03 & - & - & - & - & - \\
\hline 5.5 .72 & 0.33 & 0.31 & 0.33 & 0.01 & - & - & - & - & - & 0.02 & + \\
\hline 19.5 .72 & 0.18 & 0.40 & 0.41 & 0.01 & - & - & - & - & - & - & - \\
\hline 9.6 .72 & $0 \cdot 24$ & 0.44 & $0 \cdot 31$ & - & - & - & - & - & - & 0.01 & + \\
\hline 23.6.72 & 0.74 & 0.25 & 0.01 & - & - & - & - & - & - & - & $T$ \\
\hline
\end{tabular}




\section{(iii) Sexual reproduction}

The genotypic frequencies of sexual and non-sexual females were determined during each of the periods of sexual reproduction (table 4). Sexual females included all females whose carapace showed any trace of ephippial modification. Non-sexual females included females with parthenogenetic eggs as well as those without eggs.

TABLE 4

Genotypic frequencies during periods of sexual reproduction

\begin{tabular}{|c|c|c|c|c|c|c|c|c|c|c|c|c|}
\hline \multirow[b]{2}{*}{ Date } & \multicolumn{4}{|c|}{ Non-sexual females } & \multicolumn{4}{|c|}{ Sexual females } & \multicolumn{4}{|c|}{ Males } \\
\hline & $n$ & MM & MF & FF & $n$ & $\mathrm{MM}$ & MF & FF & $n$ & MM & MF & FF \\
\hline $20 \cdot 5 \cdot 70$ & 70 & 0.33 & $0 \cdot 46$ & 0.21 & 72 & 0.24 & 0.52 & $0 \cdot 24$ & - & -. & - & - \\
\hline $\begin{array}{r}30 \cdot 9 \cdot 70 \\
28.10 .70\end{array}$ & 388 & $0 \cdot 05$ & $0 \cdot 67$ & $0 \cdot 28$ & 42 & $0 \cdot 19$ & 0.67 & $0 \cdot 14$ & 一 & - & - & - \\
\hline 12.11 .70 & 102 & 0.04 & $0 \cdot 71$ & $0 \cdot 25$ & 37 & 0.08 & 0.81 & 0.11 & - & - & - & - \\
\hline 23.11 .70 & 113 & $0 \cdot 03$ & $0 \cdot 67$ & $0 \cdot 3$ & 30 & - & 0.73 & $0 \cdot$ & - & - & - & - \\
\hline $9 \cdot 12 \cdot 70$ & 121 & $0 \cdot 04$ & 0.75 & $0 \cdot 2$ & 22 & 0.09 & 0.59 & $0 \cdot 3$ & - & - & - & - \\
\hline 28.12 .70 & 130 & 0.04 & $0 \cdot 70$ & $0 \cdot 26$ & 38 & $0 \cdot 03$ & 0.51 & 0.46 & - & - & - & - \\
\hline $6 \cdot 1 \cdot 71$ & 140 & $0 \cdot 03$ & 0.59 & $0 \cdot 3$ & 48 & $0 \cdot 04$ & $0 \cdot 42$ & 0.5 & - & 一 & - & - \\
\hline 27.5 .71 & 149 & 0.01 & 0.72 & $0 \cdot 2$ & 12 & - & - & 1 . & 48 & $0 \cdot 02$ & 0.52 & 0.46 \\
\hline $9 \cdot 6 \cdot 71$ & 122 & $0 \cdot 02$ & 0.7 & 0. & 58 & - & $0 \cdot 1$ & 0 . & 48 & $0 \cdot 02$ & 0.56 & $0 \cdot 42$ \\
\hline 21.6 .71 & 123 & $0 \cdot 02$ & 0.79 & $0 \cdot 19$ & 50 & - & $0 \cdot 46$ & 0.5 & 46 & 一 & 0.77 & $0 \cdot 23$ \\
\hline $6 \cdot 7 \cdot 71$ & 142 & $0 \cdot 01$ & 0.77 & $0 \cdot 22$ & 36 & - & 0.94 & $0 \cdot 0$ & 48 & - & 0.85 & $0 \cdot 15$ \\
\hline $29 \cdot 12 \cdot 71$ & 142 & - & 0.44 & 0.56 & 35 & - & 0.85 & $0 \cdot 15$ & - & - & - & - \\
\hline 17.1 & 132 & - & 0.4 & 0 . & 26 & - & $0 \cdot \varepsilon$ & $0 \cdot 1$ & - & - & - & - \\
\hline 5.5 & 144 & 0.01 & $0 \cdot 2$ & 0. & 24 & 0.04 & 0.4 & 0. & - & - & - & - \\
\hline $9 \cdot 6 \cdot 72$ & 142 & - & $0 \cdot 16$ & $0 \cdot 84$ & 31 & - & 0.29 & 0.71 & - & - & - & - \\
\hline
\end{tabular}

Only one sample was analysed during the sexual period in early 1970 and no difference in genotypic frequencies between the sexual and nonsexual females was detected $(P>0 \cdot 40)$. Large differences were observed, however, during the spring of 1971. From the end of May until late June the FF homozygotes were more abundant among sexual than non-sexual females $(P<0 \cdot 001)$. This excess was less marked in the later samples, and by early June the FF homozygotes were under-represented among sexual females $(\mathrm{P}<0 \cdot 05)$. The genotypic frequencies of the males showed a similar trend. FF homozygotes were most abundant in the first sample and became less frequent in the later samples. In May 1972 the MF heterozygotes were significantly more frequent among the sexual females $(P<0 \cdot 05)$, but in the June sample the excess of MF heterozygotes was not significant $(\mathrm{P}>0 \cdot 10)$.

Genotypic frequencies of sexual and non-sexual females were significantly different during the fall and winter of 1970. From late September until mid-November the FF genotype was less frequent among the sexual than the non-sexual females $(\mathrm{P}<0.02)$, while the MM genotype was overrepresented among sexual females $(\mathrm{P}<0 \cdot 01)$. In the later samples the frequencies of the MM and MF genotypes decreased among sexual females while the frequency of the FF genotype increased. During late December and January the FF homozygote was more frequent $(\mathrm{P}<0.02)$ and the MF heterozygote less frequent $(\mathrm{P}<0.05)$ among the sexual than the non-sexual females. In 1971 sexual females were absent during the autumn and 
appeared only in the late December sample and in January 1972. The MF heterozygotes were over-represented $(\mathrm{P}<0.001)$ among the sexual females in these samples.

\section{(iv) Parthenogenetic reproduction}

After August 1970 the genotype of each individual was related to the number of eggs the individual had produced, by analysing females with differing brood sizes separately. When fewer than 30 per cent of the females were carrying parthenogenetic eggs (see table 3), a supplementary sample of at least 48 reproductive females was analysed. The females in this subsample were a random sample of the reproductive females present in the population. Absolute fecundities of the genotypes were calculated by dividing the total number of eggs carried by the individuals of one genotype by the total number of individuals of that genotype present in the sample. When a supplementary sample was analysed, the fecundity values were restored to their proper magnitudes.

Large differences in absolute fecundity from one month to another were obvious for all the genotypes (Table 5). Of more interest were the

TABLE 5

Genotypic fecundities

\begin{tabular}{|c|c|c|c|c|c|c|}
\hline \multirow[b]{2}{*}{ Date } & \multicolumn{3}{|c|}{ Absolute fecundities } & \multicolumn{3}{|c|}{ Relative fecundities* } \\
\hline & MM & MF & FF & MM & MF & FF \\
\hline August 1970 & $1 \cdot 49$ & $2 \cdot 04$ & $1 \cdot 10$ & $0 \cdot 89$ & $1 \cdot 72$ & 0.56 \\
\hline September & 0.85 & $2 \cdot 12$ & $1 \cdot 45$ & $0 \cdot 44$ & $1 \cdot 56$ & 0.71 \\
\hline October & 0.84 & $1 \cdot 13$ & $1 \cdot 17$ & 0.74 & 0.99 & $1 \cdot 06$ \\
\hline November & 1.47 & $2 \cdot 82$ & $2 \cdot 68$ & 0.53 & $1 \cdot 12$ & 0.96 \\
\hline December & - & $0 \cdot 32$ & $0 \cdot 22$ & - & $1 \cdot 72$ & 0.74 \\
\hline January 1971 & - & $1 \cdot 27$ & $0 \cdot 50$ & - & $2 \cdot 07$ & $0 \cdot 39$ \\
\hline February & - & $3 \cdot 77$ & $3 \cdot 58$ & - & 0.98 & $0 \cdot 87$ \\
\hline March & - & $20 \cdot 62$ & $18 \cdot 35$ & - & $1 \cdot 13$ & 0.90 \\
\hline April & - & $3 \cdot 12$ & $3 \cdot 23$ & - & 1.00 & $1 \cdot 05$ \\
\hline May & - & $6 \cdot 38$ & $6 \cdot 27$ & - & 1.03 & 0.98 \\
\hline June & - & $2 \cdot 74$ & $1 \cdot 63$ & - & $1 \cdot 54$ & 0.58 \\
\hline July & - & 0.06 & $0 \cdot 10$ & - & 0.59 & 1.68 \\
\hline August & - & $25 \cdot 66$ & $27 \cdot 17$ & - & $0 \cdot 94$ & 1.06 \\
\hline September & - & $7 \cdot 56$ & $8 \cdot 95$ & - & $0 \cdot 85$ & $1 \cdot 18$ \\
\hline October & - & $13 \cdot 37$ & $17 \cdot 70$ & - & $0 \cdot 76$ & $1 \cdot 33$ \\
\hline November & - & $15 \cdot 52$ & $19 \cdot 40$ & - & $0 \cdot 81$ & $1 \cdot 26$ \\
\hline December & - & $15 \cdot 71$ & $24 \cdot 49$ & - & $0 \cdot 65$ & 1.58 \\
\hline January 1972 & - & $26 \cdot 77$ & $32 \cdot 24$ & - & 0.83 & $1 \cdot 20$ \\
\hline February & - & $25 \cdot 21$ & $27 \cdot 63$ & - & 0.92 & 1.09 \\
\hline March & - & $22 \cdot 06$ & $28 \cdot 83$ & - & 0.77 & $1 \cdot 30$ \\
\hline April & - & $14 \cdot 49$ & $19 \cdot 06$ & - & 0.74 & 1.35 \\
\hline May & - & $2 \cdot 88$ & $3 \cdot 39$ & - & $0 \cdot 85$ & $1 \cdot 18$ \\
\hline June & - & $2 \cdot 20$ & $1 \cdot 85$ & - & $1 \cdot 19$ & 0.84 \\
\hline
\end{tabular}

* Relative to the frequency weighted mean fecundity of the other two genotypes.

genotypic differences in fecundity observed on several occasions. These differences were not consistent from one month to another. For example, during August and September of 1970 the MF genotype had a higher fecundity than the other genotypes, while in late 1971 and early 1972 the FF genotype had the higher fecundity. 
Such fecundity differences could have derived from two sources. Females of one genotype could have produced more eggs per reproductive female or the proportion of females with eggs could have been higher for one genotype. This latter proportion has been termed the reproductive ratio.

In most instances the reproductive ratios of the genotypes were similar (Table 6), but the MF genotype had a higher reproductive ratio $(\mathrm{P}<0.05)$

TABLE 6

Reproductive ratios and mean brood sizes of $M D H$ genotypes

\begin{tabular}{|c|c|c|c|c|c|c|}
\hline \multirow[b]{2}{*}{ Date } & \multicolumn{3}{|c|}{ Reproductive ratios } & \multicolumn{3}{|c|}{ Mean brood sizes \pm S.E. } \\
\hline & MM & MF & FF & MM & MF & FF \\
\hline August 1970 & $0 \cdot 37$ & 0.54 & $0 \cdot 29$ & $4 \cdot 25 \pm 0 \cdot 48$ & $3 \cdot 74 \pm 0 \cdot 15$ & $3 \cdot 85 \pm 0 \cdot 29$ \\
\hline September & $0 \cdot 19$ & 0.55 & 0.40 & $5 \cdot 00 \pm 0.83$ & $3 \cdot 76 \pm 0 \cdot 13$ & $3 \cdot 85 \pm 0.27$ \\
\hline October & $0 \cdot 19$ & $0 \cdot 26$ & $0 \cdot 27$ & $4 \cdot 00 \pm 0 \cdot 50$ & $4 \cdot 04 \pm 0 \cdot 17$ & $3.92 \pm 0.22$ \\
\hline November & $0 \cdot 18$ & 0.37 & $0 \cdot 32$ & $6 \cdot 00 \pm 3 \cdot 01$ & $7 \cdot 81 \pm 0.26$ & $8 \cdot 13 \pm 0 \cdot 42$ \\
\hline December & - & $0 \cdot 05$ & 0.06 & - & $6 \cdot 60 \pm 0 \cdot 60$ & $6 \cdot 00 \pm 1 \cdot 40$ \\
\hline January 1971 & - & $0 \cdot 14$ & 0.06 & - & $8.97 \pm 0.91$ & $8.57 \pm 1.93$ \\
\hline February & - & $0 \cdot 45$ & 0.48 & - & $8 \cdot 87 \pm 0.68$ & $7 \cdot 76 \pm 0.99$ \\
\hline March & - & $0 \cdot 90$ & 0.91 & - & $23 \cdot 76 \pm 1 \cdot 15$ & $20 \cdot 05 \pm 1 \cdot 15$ \\
\hline April & - & $0 \cdot 63$ & 0.70 & - & $5 \cdot 00 \pm 0.33$ & $4 \cdot 77 \pm 0 \cdot 35$ \\
\hline May & - & $0 \cdot 86$ & 0.90 & - & $7 \cdot 39 \pm 0 \cdot 19$ & $7 \cdot 00 \pm 0 \cdot 30$ \\
\hline June & - & 0.50 & $0 \cdot 32$ & - & $5 \cdot 38 \pm 0 \cdot 18$ & $5 \cdot 19 \pm 0 \cdot 28$ \\
\hline July & - & $0 \cdot 035$ & 0.04 & - & $1 \cdot 63 \pm 0 \cdot 11$ & $2 \cdot 50 \pm 0 \cdot 42$ \\
\hline August & - & 0.92 & $0 \cdot 94$ & 一 & $28 \cdot 75 \pm 1 \cdot 33$ & $29 \cdot 57 \pm 1 \cdot 82$ \\
\hline September & - & 0.89 & 0.95 & - & $8.47 \pm 0.27$ & $9 \cdot 14 \pm 0 \cdot 43$ \\
\hline October & - & 0.76 & 0.77 & - & $17 \cdot 36 \pm 0 \cdot 68$ & $22 \cdot 88 \pm 0 \cdot 86$ \\
\hline November & - & 0.76 & 0.75 & - & $20 \cdot 11 \pm 0.95$ & $26 \cdot 95 \pm 1 \cdot 19$ \\
\hline December & - & $0 \cdot 63$ & 0.75 & - & $24 \cdot 79 \pm 1 \cdot 14$ & $33 \cdot 03 \pm 1.57$ \\
\hline January 1972 & - & $0 \cdot 90$ & 0.85 & - & $29 \cdot 71 \pm 1 \cdot 17$ & $37.93 \pm 1.31$ \\
\hline February & - & 0.96 & 0.98 & - & $29 \cdot 84 \pm 1 \cdot 11$ & $32 \cdot 13 \pm 0 \cdot 91$ \\
\hline March & - & 0.92 & 0.86 & - & $23.92 \pm 1 \cdot 53$ & $33.53 \pm 1.62$ \\
\hline April & - & $0 \cdot 88$ & $0 \cdot 86$ & - & $16.47 \pm 0.97$ & $22 \cdot 10 \pm 1 \cdot 10$ \\
\hline May & - & $0 \cdot 64$ & 0.75 & - & $4 \cdot 31 \pm 0 \cdot 32$ & $4.54 \pm 0 \cdot 13$ \\
\hline June & - & $0 \cdot 54$ & $0 \cdot 50$ & - & $4 \cdot 16 \pm 0 \cdot 23$ & $3 \cdot 81 \pm 0 \cdot 14$ \\
\hline
\end{tabular}

during August and September 1970 and during January and June 1971. Significant differences in mean brood size were observed on eight occasions, during March $(\mathrm{P}<0.05)$ and July $(\mathrm{P}<0.01)$ of 1971 and again almost continuously from October 1971 until April 1972 ( $P<0.01$ with the exception of February). On seven of these occasions it was the FF genotype which had the larger brood size. The sustained difference in mean brood size observed in late 1971 and early 1972 could have resulted either from the FF homozygotes producing a few eggs more per individual than the MF heterozygotes or from a more basic difference between the two genotypes in the distribution of brood sizes. To investigate this, brood sizes were arbitrarily divided into five classes and the frequency of a given egg class among FF individuals was divided by its frequency among MF individuals to give a simple index of the relative frequency of each egg class among the two genotypes (Table 7). During August of 1971 the proportions of the FF and MF females among each of the egg classes were relatively similar, but in September there was a clear indication that a higher proportion of $\mathrm{FF}$ than $\mathrm{MF}$ individuals were carrying broods of more 
than nine eggs. In the October and November samples the FF genotypes became more frequent with increasing brood size, and during November the FF genotype was more than 30 times as frequent among the 40-109 egg class as among the 1-9 class. In the following months a bi-modality in egg distribution became apparent with the FF genotype being more abundant than the MF genotype among individuals with both the largest and smallest egg numbers.

\section{(v) Difficulties in the interpretation of fecundity differences}

Parthenogenetic offspring were normally female, but during periods of sexual reproduction some parthenogenetic eggs developed into males. At such times the fecundity values may be poor estimates of the production of female progeny, particularly if genotypic differences in male production regularly occur as in the spring of 1971 (table 4 ).

TABLE 7

Ratio of FF to MF genotypes among five egg classes

\begin{tabular}{|c|c|c|c|c|c|}
\hline \multirow[b]{2}{*}{ Date } & \multicolumn{5}{|c|}{ Brood size } \\
\hline & $1-9$ & $10-19$ & $20-29$ & $30-39$ & $40-109$ \\
\hline August 1971 & $1 \cdot 27$ & $1 \cdot 15$ & $0 \cdot 85$ & $1 \cdot 16$ & 1.04 \\
\hline September & $0 \cdot 78$ & 1.40 & - & - & - \\
\hline October & 0.47 & 0.67 & $1 \cdot 14$ & $3 \cdot 66$ & $4 \cdot 22$ \\
\hline November & $0 \cdot 19$ & 1.00 & 0.81 & $2 \cdot 01$ & 6.57 \\
\hline December & 1.67 & $0 \cdot 36$ & $0 \cdot 84$ & $0 \cdot 68$ & $8 \cdot 65$ \\
\hline January 1972 & $0 \cdot 70$ & $0 \cdot 33$ & $0 \cdot 70$ & $1 \cdot 10$ & 1.95 \\
\hline February & $2 \cdot 50$ & $1 \cdot 11$ & $0 \cdot 51$ & $1 \cdot 07$ & $2 \cdot 14$ \\
\hline March & $1 \cdot 35$ & 0.65 & 0.85 & 1.64 & 1.72 \\
\hline April & $1 \cdot 12$ & 0.52 & 0.96 & $1 \cdot 82$ & 1.48 \\
\hline
\end{tabular}

Egg counts were an accurate assessment of fecundity only so long as all eggs developed into young. In most samples egg mortality was negligible, but during early 1971 parthenogenetic egg breakdown was observed. Often entire broods of eggs deteriorated, but more frequently a few eggs developed normally while the rest died. The extent of egg mortality was estimated by comparing the number of living eggs with the total number of eggs present. No mortality was noted in the January or early February samples, but 83 per cent of the parthenogenetic eggs had deteriorated in the late February sample, and 46 per cent of the eggs in the early March sample were dead. As the Harlton population had been largely non-reproductive during January 1971, it was apparently the broods which had been produced in February which died. Egg mortality might have been the result of insufficient embryonic food supplies. Developing embryos normally shed the egg membrane early in development (Green, 1954), but during the period of egg breakdown individuals were found within the membrane with well-developed limbs and a single black eyespot.

Egg counts made during February and March of 1971 did not include moribund eggs. Estimates of relative fecundities during this period should be valid as genotypic differences in the susceptibility to egg mortality were not observed. 
(vi) Correlation between fecundity and frequency

If the fecundity differences led to the changes in frequency, then two relationships should be apparent upon comparing the fecundity and frequency data for each genotype. Firstly, the frequency of a genotype should change only if the genotypic fecundity differs from the weighted mean fecundity of the other genotypes (table 5) and secondly, changes in genotypic frequencies should lag behind fecundity differences as the embryonic Daphnia require from 2 to 5 weeks to reach maturity. The co-ordinate plot of fecundity and frequency values for the FF genotype reveal the frequent occurrence of such associations (fig. 1). The low fecundity of FF genotype

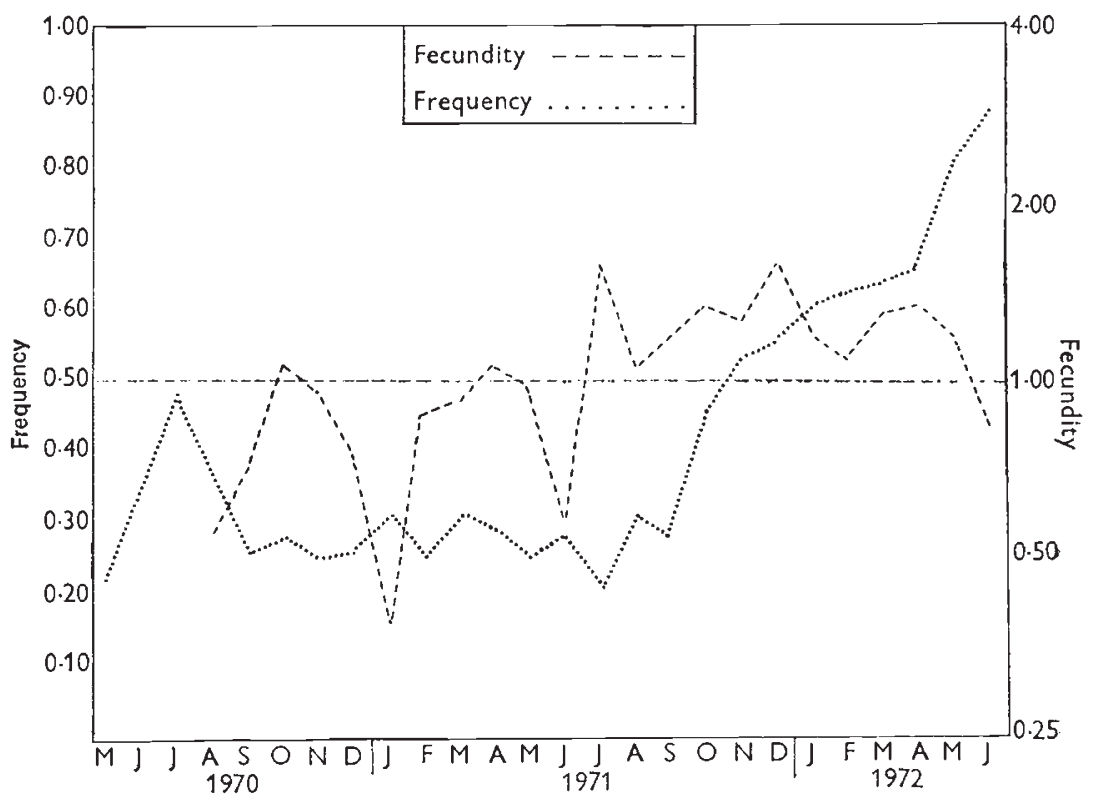

FIG. 1.-Fecundity and frequency of the MDH-FF genotype.

during August 1970 was followed by a decline in the frequency of the genotype during September. From February until May 1971 FF fecundity remained close to 1.00 and genotypic frequencies remained stable during this period and throughout June. The high fecundity of the FF genotype from October 1971 until May 1972 was accompanied by a rise in the frequency of the genotype which extended until June. The generality of these relationships was confirmed by the highly significant correlation $\mathrm{r}=0.61, \mathrm{P}<0.01$ ) between $\log _{e}$ fecundity in month $x$ and $\log _{e}$ of the change in genotypic frequency one month later, $\frac{\text { Freq }(x+1)}{\text { Freq }(x)}$. A similar correlation was observed for the MF genotype $(r=0.55, \mathrm{P}<0.01)$. The same relationship appeared to exist for the MM genotype as the fecundity of the genotype was well below 1.00 from August until December of 1970, a period when the genotype decreased in frequency. 


\section{Discussion}

The large changes of genotypic frequencies which occurred in the Harlton population were almost certainly not the result of random drift, for frequencies were relatively stable during the conspicuous minima in population size, while major changes in frequency occurred when population density was high. Genotypic differences in both parthenogenetic and sexual egg production were observed in many samples. As reproductive phenotype is affected by age (Green, 1954), differing genotypic age structures could have been the cause of these differences. The period of high FF fecundity during late 1971 and early 1972 followed a population collapse which could have caused a sampling error in genotypic age structures. It is more difficult, however, to apply such an explanation to the recurrent reproductive advantages enjoyed by the MF genotype. Moreover, sexual egg production appeared on several occasions to be related to genotypic fitness. In the autumn of 1970, when it underwent a considerable reduction in frequency, the MM genotype was over-represented among sexual females. Similarly, during late 1971 and early 1972 the MF genotype was over-represented among sexual females at the same time as it was decreasing in frequency. These observations suggested that the reproductive differences resulted from physiological differences between genotypes rather than differing genotypic age structures. In populations such as Daphnia which are repeatedly faced with large changes in food abundance, MacArthur and Wilson (1967) have argued that natural selection will favour the development of $r$ and $K$ selected genotypes. In the present study the MF genotype showed possible evidence of $K$ selection for it produced more progeny than the other genotypes when food was scarce. In contrast, the FF genotype appeared to produce more progeny when food was abundant, possible evidence of $r$ selection.

The differences in genotypic fecundities were strongly correlated with the changes in genotypic frequencies. It is important to recognise that the observed correlation underestimates the actual association between the two values. Complete correlation would exist only if the absolute change in genotypic frequency was determined by the magnitude of the measured fecundity differential. In fact, however, change in genotypic frequencies will be determined by the proportion of newly recruited adults present, as well as by the divergence in genotypic frequencies between the newly recruited and old members of the adult population. Any factor which alters the turnover rate of the adult population will alter the effect of a given fecundity differential on genotypic frequencies. High absolute reproductive rates and high temperatures ensure that new recruits will greatly outnumber the older members of the population. Conversely, decreases in temperature increase adult lifespan while slowing the maturation of immatures (MacArthur and Baillie, 1929) and as a result act to reduce the turnover rate of the adult population. Such an effect can be seen clearly in the present results. During late 1971 and early 1972 the FF genotype maintained a relatively stable fecundity advantage, yet increase in FF frequency was minimal during the winter months. Observations such as this indicate that genotypic differences in parthenogenetic egg production were of paramount importance in determining the changes in genotypic frequencies observed at Harlton. These observations further 
suggest that genotypic differences in fitness components such as developmental rate were unimportant. Such a result accords with Lewontin's (1965) theoretical conclusion that variation in egg production is likely to represent the major source of variation in $r_{m}$.

The evidence of ecological differences between genotypes accords with a growing body of data which suggest that populations of cyclical parthenogens ordinarily consist of an array of ecologically differentiated genotypes. King (1972) has, for example, shown $r_{m}$ differences between apparently conspecific clones of the rotifer Euchlanis dilatata, while Fraser (1972) observed similar differences between biotypes of the pea aphid, Acyrthosiphon pisum. Sophisticated analysis of population growth in such organisms clearly requires consideration of genetic variation. Conversely, of course, detailed investigation of the selective basis of changes in genotypic frequencies must be related to ecological parameters. Extended study of natural populations coupled with an experimental analysis of clone characteristics in controlled environments should determine if the large changes in genotypic frequencies characteristic of permanent populations of Daphnia magna are triggered by environmental changes or by the synthesis of novel, adaptively superior clones.

Acknowledgments.-I am particularly grateful to R. D. Ward for many helpful discussions and for his assistance with the collection of material. I would like as well to thank Professor J. M. Thoday and Dr J. B. Gibson for their interest in the project. The support of a Commonwealth Scholarship during the research programme and a Rutherford Scholarship and a University of Sydney post-doctoral fellowship during the preparation of the manuscript is gratefully acknowledged.

\section{REFERENCES}

DOBZHANSKY, T. H., LEWONTIN, R. C., AND PAVlosky, o. 1964. The capacity for increase in chromosomally polymorphic and monomorphic populations of Drosophila pseudoobscura. Heredity, 19, 597-614.

FRASER, B. D. 1972. Population dynamics and recognition of biotypes in the pea aphid. Can. Ent., 104, 1729-1733.

green, F. 1954. Size and reproduction in Daphnia magna. Proc. Zool. Soc. London, 124, 535-545.

HEBERT, P. D. N. 1973. Phenotypic variability of lactate dehydrogenase in Daphnia magna. 7. Exp. Zool., 186, 33-38.

HEBERT, P. D. N. 1974. Enzyme variability in natural populations of Daphnia magna. II. Genotypic frequencies in permanent populations. Genetics (in press).

HEBERT, P. D. N., AND WARD, R. D. 1972. Inheritance during parthenogenesis in Daphnia magna. Genetics, 71, 639-642.

HEBERT, P. D. N., WARD, R. D., AND GIBSON, J. B. 1972. Natural selection for enzyme variants among parthenogenetic Daphnia magna. Genet. Res. 19, 173-176.

KING, c. E. 1972. Adaptation of rotifers to seasonal variation. Ecology, 53, 408-418.

LEWONTIN, R. C. 1965. Selection for colonizing ability. In The Genetics of Colonizing Species, Ed. H. G. Baker and G. L. Stebbins. Academic Press, New York.

MACARTHUR, J. W., AND BAILLIE, w. H. T. 1929. Metabolic activity and duration of life. I. Influence of temperature on longevity in Daphnia magna. F. Exp. Zool., 53, 221-242. macarthur, R. H., AND Wilson, E. o. 1967. The Theory of Island Biogeography. Princeton University Press, Princeton.

slobodkin, L. B. 1954. Population dynamics in Daphnia obtusa. Kutz. Ecol. Monogr., 24, 69-88.

SOKAL, R. R., AND SONLEITNER, F. J. 1968. The ecology of selection in hybrid populations of Tribolium castaneum. Ecol. Monogr., 38, 345-379. 